\title{
Should I irradiate with computed tomography or sedate for magnetic resonance imaging?
}

\author{
Michael J. Callahan ${ }^{1}$ (D) Joseph P. Cravero ${ }^{2}$ \\ Received: 30 October 2020 / Revised: 3 January 2021 / Accepted: 25 January 2021 / Published online: 12 March 2021 \\ (C) The Author(s), under exclusive licence to Springer-Verlag GmbH, DE part of Springer Nature 2021
}

\begin{abstract}
In the context of pediatric cross-sectional imaging, the risk of ionizing radiation for CT and the potential adverse effects associated with sedation/anesthesia for MRI continue to provoke lively discussions in the pediatric literature and lay press. This is particularly true for issues relating to the risks of ionizing radiation for $\mathrm{CT}$, which has been a topic of discussion for nearly two decades. In addition to understanding these potential risks and the importance of minimizing individual pediatric patient exposure to ionizing radiation, it is equally important for radiologists to be able to frame these risks with respect to the potential for adverse outcomes associated with the use of anesthesia for cross-sectional imaging in the pediatric population. Notably, before such risks can be estimated and compared, one should always consider the potential utility of each imaging modality for a given diagnosis. If one cross-sectional imaging modality is likely to be far superior to the other for a specific clinical question, every effort must be made to safely image the child, even if sedation/anesthesia is required.
\end{abstract}

Keywords Anesthesia $\cdot$ Children $\cdot$ Computed tomography $\cdot$ Ionizing radiation $\cdot$ Magnetic resonance imaging $\cdot$ Risks $\cdot$ Sedation

\section{Introduction}

The question "Should I irradiate with CT or sedate for MRI?" is largely rhetorical, and it is a multifaceted topic that does not allow for a specific answer for the pediatric population at large. Risks of carcinogenesis relating to ionizing radiation have traditionally been population-based and have been estimated and predicted with the linear-no-threshold (LNT) hypothesis, which was initially founded upon atomic bomb survivor data. The LNT hypothesis specifies that biological injury resulting from exposure to ionizing radiation is directly proportional to dose in a linear fashion [1-3]. As such, according to the LNT hypothesis there is no dose threshold whereby there is no risk, even for a single CT examination. Alternatively, some groups including the American

Michael J. Callahan

michael.callahan@childrens.harvard.edu

1 Department of Radiology, Boston Children's Hospital, Harvard Medical School, 300 Longwood Ave., Boston, MA 02115, USA

2 Department of Anesthesiology, Boston Children's Hospital, Harvard Medical School, Boston, MA, USA
Association of Physicists in Medicine suggest that the development of carcinogenesis requires a cumulative threshold of at least 50 millisieverts $(\mathrm{mSv})$ for a single $\mathrm{CT}$ examination and $100 \mathrm{mSv}$ for multiple CT examinations [4-8], both of which are orders of magnitude higher than a typical CT scan on a modern scanner. In contrast to the LNT hypothesis, this would suggest that there is no statistically measureable increased risk of carcinogenesis for a single CT exam.

Large-scale data for perioperative anesthesia risks in children have been published [9-12]; however, immediate risks of anesthesia must also be considered on a case-by-case basis. The actual risk to any individual child is dependent on his or her current health status and comorbid conditions. Longerterm neurocognitive risks of anesthesia have also been described [9-12], but the data on this topic are not definitive because the potentially negative effects on childhood learning by anesthetics might in fact be caused by confounding variables, most notably the underlying illness that necessitated anesthesia for surgical intervention [13-15].

Providing safe and effective pediatric imaging is a challenging task, particularly at tertiary-care medical centers where some of the most acutely ill and most complex patients require high-quality cross-sectional imaging on a regular basis. To meet the needs of our patients, effective pediatric imaging necessitates an experienced multidisciplinary team to 
foster a well-organized and efficient imaging environment to ensure the safety and well-being of our patients, as well as our technologists, nurses, anesthesiologists, radiologists and hospital support staff.

Computed tomography and MRI are noninvasive imaging techniques that represent the cornerstone of reliable and reproducible cross-sectional diagnostic imaging in the pediatric population, and technology continues to evolve for each modality. Until recently, both CT and MRI commonly required sedation or anesthesia for most infants, young children and developmentally delayed individuals. However, recent advances in multidetector CT technology have enabled much more rapid imaging techniques, on the order of $1 \mathrm{~s}$ or less for an entire diagnostic CT examination. In contrast, even the shortest of MRI protocols still require several minutes of imaging time (and many extending for an hour or more), requiring the use of sedation or anesthesia in pediatric patients who are unable to voluntarily hold still in a noisy, confined environment, or breath-hold on command. We believe that the development of ultrafast $\mathrm{CT}$ imaging (and a resultant decreased requirement for sedation/anesthesia for pediatric CT) [16] allows one to reconsider the risks of CT radiation against the risks of sedation/anesthesia for MRI. As such, a comparison of these risks has been highlighted in the pediatric literature [17-19]. However, before these risks can be estimated and compared, one must always assess the sensitivity and specificity of each imaging modality for a given diagnosis. If one modality is far superior to the other for a specific clinical question, every effort must be made to safely image the child with the most appropriate imaging modality if clinically warranted.

\section{Risks of ionizing radiation from computed tomography}

The potential risks of carcinogenesis related to ionizing radiation exposure from medical imaging have been comprehensively evaluated in the literature [20-23]. Risk estimates have primarily been derived from the linear-no-threshold (LNT) hypothesis, which has driven regulatory policies [19] and is accepted by many as the best model for risk assessment. Several age-based estimates were initially published by Brenner et al. [1], stating that at least 600,000 abdominal and head CT examinations were performed annually in the United States (2001) on children younger than 15 years and, of these individuals, a rough estimate is that approximately $500(8.3 / 10,000)$ would ultimately die from a cancer attributable to the radiation from CT. In a more recent retrospective cohort study in children using Poisson relative risk models, Pearce et al. [24] estimated that for children younger than 10 years, within 10 years after a first head CT scan, one excess case of leukemia and one excess case of brain tumor would occur per 10,000 individuals. Mathews et al. [25] identified a cohort of Australian patients younger than 19 years who were exposed to a CT scan greater than 1 year prior to a diagnosis of cancer. They estimated an absolute excess cancer incidence rate of 9.4 per 100,000 person years when exposed to an average of $4.5 \mathrm{mSv}$ [25].

\section{Risks of anesthesia for magnetic resonance imaging}

Anesthesia risks in pediatric patients can be sub-classified into the immediate risks that can occur during the active administration of drugs, and the long-term neurocognitive risks. Immediate risks generally relate to airway management, ventilation/oxygenation and hemodynamic stability. Largescale perioperative data broadly suggest that the immediate risks of anesthesia are approximately 1 in 10,000 for death, cardiac arrest or similar severe adverse events [9, 26, 27]. Specific risks of MRI with sedation/anesthesia have been described in the pediatric anesthesia literature, citing that the extent of risk for general anesthesia varies with patient age and underlying pathology [17]. In particular, populations at a higher risk of adverse events and pulmonary complications include children younger than 1 year and people with an American Society of Anesthesiologists (ASA) status score of $\geq 3$ [28]. Preterm infants are reported to have a significant risk of apneic events after anesthesia [29]. Developmentally delayed children are three times more likely to experience hypoxia during deep sedation/anesthesia relative to developmentally normal children [30]. Risks are even more prevalent in children with congenital cardiac disease undergoing MRI with sedation/anesthesia, with published adverse event values as high as $10.4 \%$ for this patient subset [31], including minor hypoxia and bronchospasm, and major pulmonary edema, severe hypotension and tachypnea. These studies highlight the concept that the immediate risk associated with sedation/anesthesia is not uniform and, in fact, varies by orders of magnitude across children with different underlying conditions.

When considering the overall (immediate) risk of anesthesia/sedation for MRI, it is important to note that the risk for adverse events is compounded by physical separation between the patient and the anesthesiologist during these studies, particularly when the child is actively being imaged in the bore of the unit [32].

Long-term neurocognitive effects of anesthesia/sedation are much less clearly documented but have become a significant consideration for many families and anesthesiologists. Multiple animal models have demonstrated that exposure to sedation/anesthesia at specific developmental epochs can lead to neuroapoptosis and demonstrable neurocognitive deterioration [33-35]. Demographic studies in humans have suggested an association between multiple anesthetic exposures at very early ages and an increase in learning difficulties later in life $[14,15]$. On the other hand, there is only one randomized 
controlled study of the developmental effects of general anesthesia, and it failed to show any adverse results from a single general anesthesia exposure in young infants [36]. It is generally agreed that repeated and longer exposures in very young children represent the highest risk situations for pediatric patients when it comes to neurocognitive outcomes [37].

Specific predictors of anesthetic exposure for pediatric MRI have been described in the recent literature, with a focus on minimizing MRI scan times. In their retrospective study, Machado-Rivas et al. [38] evaluated a cohort of pediatric patients requiring anesthesia for MRI examinations and determined that children requiring propofol anesthesia received a higher dose for multiple body part examinations relative to single body part examinations. Additional predictors of longer scan time for single body part examinations included higher patient ASA classification, an oncological diagnosis, use of a 1.5-T magnet, and intravenous contrast administration [38]. The authors concluded that pediatric MRI anesthetic exposure can be quantified and potentially predicted based on the type of imaging to be performed and patient clinical variables, which might serve as a baseline for future efforts to minimize anesthetic doses for pediatric MRI [38].

In addition to the risks of sedation for certain pediatric MR patients, MRI has its own inherent risks for caregivers, including the potential effects of electromagnetic fields and highpowered radiofrequency coils. Potential safety risks for patients and personnel include, but are not exclusive to, projectile injury, skin burns, interference with implants and other medical devices, and acoustic injuries [39]. These risks are quite small if standard MRI screening and safety procedures are followed.

Although of secondary importance in the setting of patient safety, it worth noting that use of anesthesia for pediatric patients undergoing MRI introduces an additional level of financial cost for anesthesia and nursing staffing. An additional recovery period is also required, which necessitates specialized patient monitoring, typically in a hospital setting. For these reasons, all other factors being equal, a CT scan performed without sedation is substantially less expensive than an MRI with sedation.

\section{COVID-19}

During the novel coronavirus disease 2019 (COVID-19) pandemic, the American College of Radiology provided initial suggestions for imaging people who have tested positive for COVID-19 and for persons under investigation for the disease, stating that practitioners should minimize the use of MRI except when absolutely necessary [40]. Restrictions have softened since the early days of the pandemic, although accommodations for patient testing and increased use of personal protective equipment (PPE) for cross-sectional imaging relating to COVID-19 continued at the time of this report.
There are no data on the potential impact of COVID-19 infection on the adverse event rate during anesthesia itself. Although many children are asymptomatic when they contract the virus, the most common symptoms encountered are upper or lower respiratory illness [41]. It is logical that children with significant respiratory illness would have an increased need for airway intervention and an increase in potential adverse events, just as they would with non-COVID-related airway disease.

Sedation and anesthesia are classified as potentially aerosol-generating procedures (AGP), largely because of the associated incidence of bag-mask ventilation, intubation and extubation. These AGPs can result in increased risk to anesthesia health care workers exposed to patients with COVID19. As such, technical changes for airway management in COVID-19 patients undergoing MRI have been described [42], including airway management in a negative pressure room and expanded use of PPE. All of these considerations change the nature of workflow for children requiring sedation/ anesthesia for MRI when they have COVID-19 infection. The location of induction and recovery might be changed, the need to transport the child through the hospital to a recovery location must be considered. Additional time is required to clean and allow air turnover in rooms where a child with COVID-19 infection has been cared for and undergone an AGP [43]. It is likely that these changes in normal procedures add to the possibility of adverse events in these cases.

Finally, although patient care is always of paramount importance, referring clinicians and radiologists must also recognize and consider the additional risks that sedation/ anesthesia exposes frontline workers and other caregivers to during a pandemic. These practical risks should be understood when weighing the risk vs. benefit of a given imaging modality.

\section{Discussion}

Comparisons of the risks of radiation from CT imaging against the risks of anesthesia for MRI have been described in the pediatric literature [17-19, 44, 45], and this discussion continues to evolve. In a modern diagnostic imaging department, it is no longer sensible for the radiologist to be responsible for sedation [46], particularly for pediatric patients with complex diseases and co-morbidities. High-quality pediatric sedation for cross-sectional imaging requires an experienced anesthesiologist who can minimize patient motion while maximizing patient safety. It is well documented that anesthesia and radiology need to be partners [47] in an effort to safely image pediatric patients in diagnostic imaging departments by identifying common problems, improving efficiency, and maximizing collegiality and transparency. 
Both radiologists and anesthesiologists have an obligation and responsibility to investigate and discuss issues relating to patient safety for each individual patient entrusted with their care. There should be an opportunity for anesthesiologists to be directly involved in the discussion with radiologists and referring physicians regarding the risks and benefits for a particular imaging test on an individual patient basis, particularly risks related to sedation/anesthesia. There is often a need to customize the imaging plan based on the inherent risk to the patient. In the interest of transparency and full disclosure, the entire gamut of these risks should be presented to patients (if age appropriate) and families to help them provide input into the decision-making process.

Although efforts are being made to decrease the use of anesthesia for MRI and decrease MR scan times, the use of sedation and anesthesia for pediatric MRI is still very common. Based on a relatively recent New York State retrospective database, anesthesia use for ambulatory MRI was required in $91 \%$ of children younger than 1 year and $84 \%$ of children ages $1-$ 6 years [48]. Given the widespread use of sedation and anesthesia for pediatric MR imaging, this remains a topic of interest for researchers. Recent advances in ultrafast MR imaging techniques are bridging the gap between $\mathrm{CT}$ and MR imaging times $[49,50]$, but it is unlikely that MRI exam times will rival the extremely rapid CT scan times in the near future.

As is true for anesthesia risks for MRI, the risks of radiation for CT are likely higher in younger patients, but immediate risks of CT for very complex and ill inpatients are fewer than those in MRI, assuming sedation or anesthesia is required for MRI and not for CT. A consideration of these risks should be accounted for when imaging studies are requested and planned for both inpatients and high-risk outpatients. For instance, the relative risk of sedation/anesthesia for a child with severe congenital heart disease or advanced malignancy might far outweigh the long-term cancer risk incurred by the radiation exposure from a CT scan.

Image Gently and Image Wisely are health care coalitions and social awareness campaigns that are dedicated to fostering safe, high-quality imaging for pediatric and adult patients worldwide [51, 52]. Their websites provide free educational materials that have been vetted by experts in imaging fields. The ultimate goal of these campaigns is to raise awareness and change practice in an effort to minimize radiation exposure to pediatric and adult patients. Similarly, SmartTots is a collaborative effort of the International Anesthesia Research Society and the United States Food and Drug Administration [53]. The mission of this initiative is to fund and coordinate research with the goal of making surgery safer for infants and children.

\section{Conclusion}

In general, most pediatric radiologists appropriately adopt a conservative stance on the use of ionizing radiation in pediatric patients, minimizing exposure whenever possible. Although minimizing exposure to ionizing radiation is of paramount importance in infants and children, we believe a similar avoidance of anesthesia in vulnerable patient populations must be considered in certain clinical situations. The risks of sedation/anesthesia for MRI in critically ill children (ASA $\geq 3$ ), premature infants, and developmentally delayed children with severe comorbidities including congenital heart disease is substantially higher than for healthy outpatients, and these risks cannot be dismissed simply to minimize exposure to ionizing radiation. Furthermore, the occupational risk that is presented by the COVID-19 pandemic requires some consideration when choosing an imaging modality for a given patient. There is a need to consider input from multiple sources, including medical specialists and family members, to formulate the most informed, shared decision for each child.

\section{Declarations}

\section{Conflicts of interest None}

\section{References}

1. Brenner DJ, Elliston CD, Hall EJ, Berdon WE (2001) Estimated risks of radiation-induced fatal cancer from pediatric CT. AJR Am J Roentgenol 176:289-296

2. Preston DL, Ron E, Tokuoka S et al (2007) Solid cancer incidence in atomic bomb survivors: 1958-1998. Radiat Res 168:1-64

3. Little MP, Wakeford R, Tawn J et al (2009) Risks associated with low doses and low dose rates of ionizing radiation: why linearity may be (almost) the best we can do. Radiology 251:6-12

4. American Association of Physicists in Medicine (2011) Position statement. Radiation risks from medical imaging procedures. http://www.aapm.org/org/policies/details.asp?id=318\&type=PP. Accessed 5 Oct 2017

5. Harvey HB, Brink JD, Frush DP (2015) Informed consent for radiation risk from $\mathrm{CT}$ is unjustified based on the current scientific evidence. Radiology 275:321-325

6. Ozasa K, Shimizu Y, Suyama A et al (2012) Studies of the mortality of atomic bomb survivors, report 14, 1950-2003: an overview of cancer and noncancer diseases. Radiat Res 177:229-243

7. Tubiana M, Feinendegen LE, Yang C, Kaminski JM (2009) The linear no-threshold relationship is inconsistent with radiation biologic and experimental data. Radiology 251:13-22

8. Hendee WR, O'Connor MK (2012) Radiation risks of medical imaging: separating fact from fantasy. Radiology 264:312-321

9. Paterson N, Waterhouse P (2011) Risk in pediatric anesthesia. Paediatr Anaesth 21:848-857

10. Cravero JP (2009) Risk and safety of pediatric sedation/anesthesia for procedures outside the operating room. Curr Opin Anaesthesiol 22:509-513

11. Morray JP (2002) Anesthesia-related cardiac arrest in children. An update. Anesthesiol Clin North Am 20:1-28

12. Gonzalez LP, Pignaton W, Kusano PS et al (2012) Anesthesiarelated mortality in pediatric patients: a systematic review. Clinics 67:381-387

13. Barton K, Nickerson JP, Higgins T et al (2018) Pediatric anesthesia and neurotoxicity: what the radiologist needs to know. Pediatr Radiol 48:31-36 
14. Wilder RT, Flick RP, Sprung J et al (2009) Early exposure to anesthesia and learning disabilities in a population-based birth cohort. Anesthesiology 110:796-804

15. Flick RP, Katusic SK, Colligan RC et al (2011) Cognitive and behavioral outcomes after early exposure to anesthesia and surgery. Pediatrics 128:e1053-e1061

16. Kino A, Zucker EJ, Honkanen A et al (2019) Ultrafast pediatric chest computed tomography: comparison of free-breathing vs. breath-hold imaging with and without anesthesia in young children. Pediatr Radiol 49:301-307

17. Masaracchia MM, Tsapakos MJ, McNulty NJ, Beach ML (2017) Changing the paradigm for diagnostic MRI in pediatrics: don't hold your breath. Paediatr Anaesth 27:880-884

18. Greenberg SB (2011) Rebalancing the risks of computed tomography and magnetic resonance imaging. Pediatr Radiol 41:951-952

19. Callahan MJ, MacDougall RD, Bixby SD et al (2018) Ionizing radiation from computed tomography versus anesthesia for magnetic resonance imaging in infants and children: patient safety considerations. Pediatr Radiol 48:21-30

20. Frush DP, Donnolly LD, Rosen NS (2003) Computed tomography and radiation risks: what pediatric health care providers should know. Pediatrics 112:951-957

21. Brody AS, Frush DP, Huda W et al (2007) Radiation risk to children from computed tomography. Pediatrics 120:677-682

22. Miglioretti DL, Johnson E, Williams A et al (2013) The use of computed tomography in pediatrics and the associated radiation exposure and estimated cancer risk. JAMA Pediatr 167:700-707

23. Brenner DJ, Hall EJ (2007) Computed tomography - an increasing source of radiation exposure. N Engl J Med 357:2277-2284

24. Pearce MS, Salotti JA, Little MP et al (2012) Radiation exposure from CT scans in childhood and subsequent risk of leukaemia and brain tumours: a retrospective cohort. Lancet 380:499-505

25. Mathews JD, Forsythe AV, Brad Z et al (2013) Cancer risk in 680,000 people exposed to computed tomography scans in childhood or adolescence: data linkage study of 11 million Australians. BMJ 346:f2360

26. Tay CL, Tan GM, Ng SB (2001) Critical incidents in paediatric anaesthesia: an audit of 10,000 anaesthetics in Singapore. Paediatr Anaesth 11:711-718

27. Murat I, Constant I, Maud'huy H (2004) Perioperative anaesthetic morbidity in children: a database of 24,165 anaesthetics over a 30month period. Paediatr Anaesth 14:158-166

28. Cravero JP, Beach ML, Blike GT et al (2009) The incidence and nature of adverse events during pediatric sedation/anesthesia with propofol for procedures outside the operating room: a report from the pediatric sedation research consortium. Anesth Analg 108:795804

29. Cote CJ, Zaslavsky A, Downes JJ et al (1995) Postoperative apnea in former preterm infants after inguinal herniorrhaphy: a combined analysis. Anesthesiology 82:809-822

30. Kannikeswaran N, Mahajan P, Sethurman U et al (2009) Sedation medication received and adverse events related to sedation for brain MRI in children with and without developmental disabilities. Paediatr Anaesth 19:250-256

31. Dorfman AL, Odegard KC, Powell AJ et al (2007) Risk factors for adverse events during cardiovascular magnetic resonance in congenital heart disease. J Cardiovasc Magn Reson 9:793-798

32. Gilk T (2006) MRI suites: safety outside the bore. Patient Safety Quality Healthcare. https://www.psqh.com/sepoct06/mrisuites. html. Accessed 15 Sep 2016

33. Rappaport B, Mellon D, Simone A et al (2011) Defining safe use of anesthesia in children. New Engl J Med 364:1387-1390

34. Jevtovic-Todorovic V, Hartman RE, Izumi Y et al (2003) Early exposure to common anesthetic agents causes widespread neurodegeneration in the developing rat brain and persistent learning deficits. J Neurosci 23:876-882

35. Young C, Jevtovic-Todorovic V, Qin YQ et al (2005) Potential of ketamine and midazolam, individually or in combination, to induce apoptotic neurodegeneration in the infant mouse brain. Br J Pharmacol 146:189-197

36. McCann ME, de Graaf JC, Dorris L et al (2019) Neurodevelopmental outcome at 5 years of age after general anaesthesia or awake-regional anesthesia in infancy (GAS): an international, multicentre, randomized, controlled equivalence trial. Lancet 393:664-677

37. McCann ME, Soriano SG (2012) General anesthetics in pediatric anesthesia: influences on the developing brain. Curr Drug Targets 13:944-951

38. Machado-Rivas F, Leitman E, Jaimes C et al (2020) Predictors of anesthetic exposure in pediatric MRI. AJR Am J Roentgenol 215: $1-9$

39. Tsai LL, Grant AK, Mortele KJ et al (2015) A practical guide to MR imaging safety: what radiologists need to know. Radiographics 35:1722-1737

40. American College of Radiology (2020) ACR guidance on COVID19 and MR use. ACR website. https://www.acr.org/ClinicalResources/Radiology-Safety/MR-Safety/COVID-19-and-MR-Use. Accessed 24 Oct 2020

41. Cai J, Xu J, Lin D et al (2020) A case series of children with 2019 novel coronavirus infection: clinical and epidemiological features. Clin Infect Dis 71:1547-1551

42. Drum E, McClung Pasqualino H, Subramanyam R (2020) Anesthesia and potential aerosol generation during magnetic resonance imaging in children with COVID-19. Paediatr Anaesth 30: 944-946

43. Wax RS, Christian MD (2020) Practical recommendations for critical care and anesthesiology teams caring for novel coronavirus (2019-nCoV) patients. Can J Anaesth 67:568-576

44. Cauldwell C (2011) Anesthesia risks associated with pediatric imaging. Pediatr Radiol 41:949-950

45. Templeton LC, Sharna R, Templeton TW (2017) There's no anesthesia like no anesthesia. Paediatr Anaesth 27:1167-1168

46. Slovis TL (2011) Sedation and anesthesia issues in pediatric imaging. Pediatr Radiol 41:514

47. Mahmoud M, Jones B, Podberesky DJ (2013) Teamwork among pediatric anesthesia and radiology providers at a large tertiary-care children's hospital: past, present and future. Pediatr Radiol 43:460 463

48. Huang YY, Ing C, Li G, Sun LS (2016) Analysis of MRI utilization in pediatric patients. J Neurosurg Anesthesiol 28:413-418

49. Kozak BM, Jaimes C, Kirsch J, Gee MS (2020) MRI techniques to decrease imaging times in children. Radiographics 40:485-502

50. Jaimes C, Gee MS (2016) Strategies to minimize sedation in pediatric body magnetic resonance imaging. Pediatr Radiol 46:916-927

51. Image Gently Alliance (2014) Image Gently campaign website. Imagegently.org. Accessed 20 Jan 2021

52. American College of Radiology, Radiological Society of North America, American Society of Radiological Technologists, American Association of Physicists in Medicine (2021) Image Wisely campaign website. Imagewisely.org. Accessed 20 Jan 2021

53. SmartTots (n.d.) Funding research to ensure pediatric anesthesia safety. Website. https://smarttots.org/. Accessed 20 Jan 2021

Publisher's note Springer Nature remains neutral with regard to jurisdictional claims in published maps and institutional affiliations. 